\title{
Design of the rectifier bridge machine design Based on PLC control
}

\author{
Lijian Zhang ${ }^{1, a}$, Junjie Wang ${ }^{2, b}$, Tingting Wang ${ }^{2, c}$ \\ ${ }^{1}$ College of Electrical Engineering, Binzhou Polytechnic,Shandong,256600,China \\ ${ }^{2}$ Bohai Piston Co., Ltd, Binzhou,Shandong,256600,China

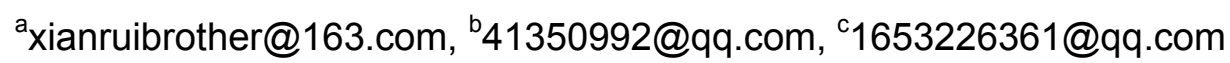

Keywords: Machine, Detection, Classification, Laser

\begin{abstract}
: this paper designed and developed a new type of rectifier bridge machine, the equipment can efficiently in automatic mode and manual mode to complete the detection and classification of the rectifier bridge, the laser marking and the pendulum work, greatly improving the sorting efficiency of the rectifier bridge. And the mechanical structure and working principle of the equipment are expounded.
\end{abstract}

\section{Introduction}

In production, China is a rectifier bridge rectifier bridge in People's Daily life and work has universal application. However, production of the rectifier bridge belongs to the labor-intensive industries, the factory inspection, marking and pendulum material such as most of the work is still rely on artificial to complete. The pendulum feeder single function, complicated operation, not completed efficient laser marking and the pendulum material work, greatly reduces the production efficiency.

His equipment is a kind of can be based on PLC and touch screen and chip high-speed pendulum feeder is the formation of the sensors, PLC, pneumatic automatic device. Accurately positioning servo stepper motor and human-computer interaction with touch screen technology, system operation in the process of all kinds of failure, can be displayed on the touch screen, and sound and light alarm. The control system has a variety of work mode choice: single track or double track mode, test mode, or demo mode, automatic mode or manual mode.

\section{Device structure and working principle}

\section{Equipment structure}

The pendulum feeder detection, laser code and realize rectifier bridge rules set material classification, etc.

Set machine body has a feeding device, inclined ramp device, orbit around shunt mobile device, orbit around device, materials plate device, PLC control, man-machine display device, laser marking device, rectifier bridge detection device, sensor and cylinder and other parts. The whole system structure is shown in figure 1. 


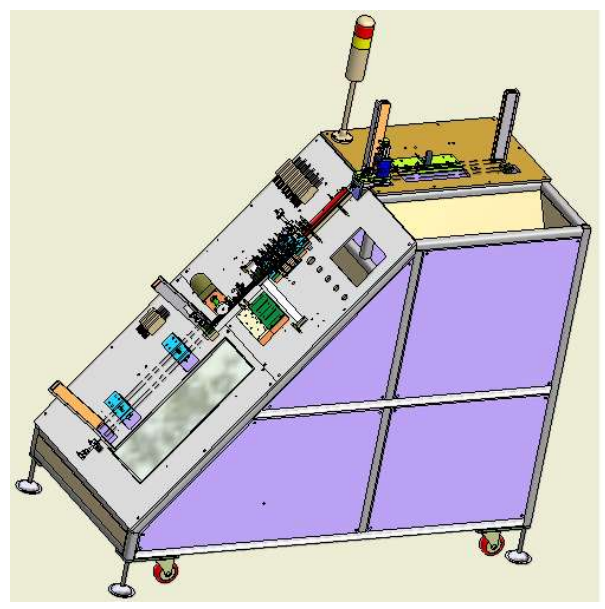

Fig.1: System assembly

\section{The design and working principle of the device}

In equipment operation, the operator manual will be equipped with new production casing rectifier bridge into the feeding device of material storage device, material storage devices can store a lot of casing. If the installation in the inclined ramp material level under the lower limit of sensors is less than rectifier bridge, and delay after a certain time, back to a horizontal position, rotating cylinder ejector sleeve cylinder will contain the casing rectifier bridge to be detected, feeding pipe jacking out of the casing in place, delay after a certain time, feeding pressure pipe casing pressure, feeding rotating cylinder rotate in place, after feeding resistance open a needle, rectifier bridge, relying on its own weight, falling to the inclined ramp device, complete automatic feeding operation of equipment.

Orbit around shunt device consists of a double cylinder drag a slider to implement. When out cylinder in place, into the slider on the left side of the rectifier bridge from the feeding chute, if dual track mode is selected, the slider on the right side into the right track the rectifier bridge; When the cylinder back in place, rectifier bridge from the feeding chute into the slider on the right side, if the choice is a dual track mode, the slider on the left side of the rectifier bridge into the left orbit. If select left orbit or right track model, rectifier bridge can only from the feeding chute into the corresponding side slide block, avoid the waste of material.

Inclined ramp device is equipped with the upper limit of material level and lower level lower limit sensor, the upper limit of material level sensor can't detect the material time more than the set value, feeding operation again rectifier bridge, to ensure that the inclined ramp device has to be testing rectifier bridge material. The material level lower limit sensor detects the rectifier bridge, and the left orbital device left material sensors is less than 1 or right track device right material sensors is less than 1, the equipment through orbit around tap device will be put into orbit around the rectifier bridge. According to the operation mode of the man-machine interface to choose, can separate the rectifier bridge rail shunt to the left or right around orbit or track. Feed opening sensor installed on the inclined ramp device can statistical sorting and the working speed of total rectifier bridge, and real-time display in man-machine interface.

Orbit around device right and left orbital and orbital device. Each track has five resistance and five sensors, test whether the material is a rectifier bridge. Resistance level 1 used for temporary orbit around from distributary device of rectifier bridge, a 2, resistance 3, drag a 4 to temporary waiting for inspection of the rectifier bridge, corresponding position is driven by the clamping test piece realizes the rectifier bridge detection, test results is divided into six categories, such as, 
electrical bad, over voltage, etc. Each track side placed three test machine, can be on the man-machine interface free combination of the three test machine working state, realize the diversification of rectifier bridge detection. PLC storage testing machine test results, use in the subsequent process. Each resistance can only staging a rectifier bridge, otherwise there will be a superposition of the rectifier bridge above can't normal inspection, and cause error classification. Through a bottom-up detection mechanism to realize rectifier bridge in the detection of orbit moving step by step. Here in three test machine to work at the same time, describe how it works. Resistance level 4 of the rectifier bridge detection, after the completion of the test machine to complete the detection of the rectifier bridge, will send the test signal EOT input to PLC, PLC, upon receipt of this signal by resistance to drag a 4 cylinder open, drag a rectifier bridge into resistance level 4 5; If the resistance of three rectifier bridge testing machine also completed the testing work, the PLC is controlled by the output resistance of 3 cylinder, open the resistance level 3, drag a rectifier bridge into resistance level 3 4; If the resistance of two rectifier bridge testing machine also completed the testing work, the PLC controlled by the output resistance 2 cylinder, open the resistance level 2, rectifier bridge into the resistance of a 3 3, the PLC open resistance 1, will drag bit the rectifier bridge into resistance level 12 ; Thus realizes the rectifier bridge from top to bottom in order, will not lead to two rectifier bridge falling at the same time.

Are mobile devices by inovance servo motor through the synchronous belt drives the sorting slide block device, which achieves the classification of the tested complete rectifier bridge work. Servo motor control steps of walking, slide block device with various of entry LieShou feed tube entry accurate registration, by changing the output frequency of the servo motor, to control the movement of the slider device speed. Tested six class complete classification of rectifier bridge, the first is the normal rectifier, the rest of the all kinds of what kind of store can have PC to set rectifier bridge, can put poor contact rectifier bridge in the second category, can also be into the third category, according to the specific requirements to decide. In addition to the first category and the follow-up work is not directly into receiving tube, the rest of the five categories corresponding to a feeding tube, receiving tube if not insert, sound and light alarm, the entire device suspended work, when receiving tube insertion, sound and light alarm, the machine normal operation. At the end of rectifier bridge into which category, on the man-machine interface can display in number and in total. When the total to set in the receiving tube, sound and light alarm, after artificial in pipe, equipment continues to work.

When the test machine to detect the normal rectifier fall into the first category, laser on the keep-off cylinder is in open state, under the laser keep-off cylinder is closed, such as laser detection, laser detection under both sensors detect the rectifier bridge, delay time, laser printers on the four pieces of rectifier bridge preset word, waiting for printing, laser on the keep-off cylinder closed, under the laser keep-off cylinder is open, four pieces of rectifier bridge in clean dust brush power slide down upon receiving tube.

When the first number in the receiving tube rectifier bridge reach a set number, PC is given the corresponding alarm prompt screen, not blocking the normal operation of the machine. Begin receiving equipment operation, the receiving tube pressure cylinder is raised, the receiving pipe jacking cylinder in a retracted state, receiving tube cylinder retracted, receiving cylinder ejector pin, will the next feeding tube replace the current feed tube, and receiving tube will launch to the temporary storage device. At this point, the discharging pipe jacking cylinder, receiving pressure cylinder pressure discharging tube, receiving tube out cylinder, receiving the ejector sleeve cylinder retracted, complete in receiving tube process.

When the machine starts, self-checking equipment first, if there is a failure, on the upper machine 
for prompt operation. If the device is normal, for cleaning operation orbit around rectifier bridge, will stay in orbit when stop rectifier bridge clean up to the sixth class discharging pipe, the sixth class rectifier bridge in the receiving tube can be classified in testing.

\section{System structure}

The structure of the part of electric control system is shown in figure 2. Whole control equipment with inovance $\mathrm{H} 2 \mathrm{U}$ - XP programmable controller as the control core, Y0, Y1, Y3 for high-speed pulse output, this equipment in Y0 mouth control servo motor work direction, to Y1 output high-speed pulse output string control the operation of the servo motor. Use absolute positioning system internal function instruction DDRVA, the instruction is according to the specified port and output pulse frequency and direction, make the servo actuator movement to specify the destination point. Special relay M8145 said Y0 pulse output stop. Special relay M8147 said Y0 pulse output monitoring (BUSY/READY). In the key parts of the equipment equipped with sensors, ladder diagram program according to the logic, complete automation equipment.

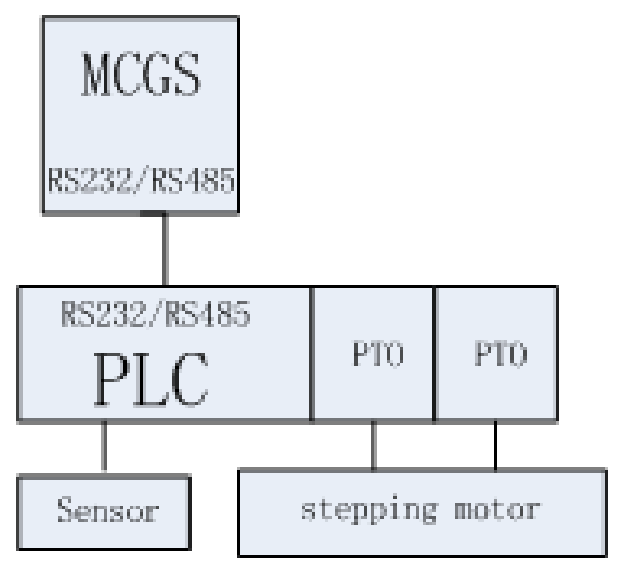

Fig. 2 control system block diagram

This system is the system a total of 61 type, 76 dots, 14 omron sensor installed. The omron MY2N - J intermediate relay to increase the anti-interference ability of the system.

\section{The basic functions}

Through the start, stop and reset three buttons control the operation of equipment. To prevent hardware failure, to achieve double redundancy Yu Jiegou, also add three buttons on touch screen. Added the servo motor control the touch screen, the picture has inching control, continuous control input box and operating frequency and operating steps, convenient debugging personnel debugging, adjust the slider device with accurate registration in the receiving port. Orbit of various types of sensors, control devices of feeding, picking up the material, classification, and so on.

\section{Conclusion}

The whole equipment structure compact, dexterity, on the premise of fully considering the technical workers operating habits, PLC is the core of the equipment, greatly reduces the production cost. Automatic pendulum material machine and laser printer with work, improve the production efficiency, reduce the operating personnel, has been widely applied in the rectifier bridge industry.

\section{Acknowledgement}

This research was financially supported by Colleges and universities in shandong province department of education scientific research and development projects. 
Project name: intravenous infusion of the control system of automatic dispensing apparatus development with item number: J14LB57.

\section{Reference}

[1] Ya-jun li. the straightness error of the data processing and program design [J]. China measurement technology,2007, 33 (3).

[2] Chen guoqiang. Based on the MATLAB precise straightness error evaluation [J]. Journal of machine tools and hydraulic, 2006 (2).

[3] Lin Xiang. Straightness error of the new algorithm and its implementation on microcomputer [J]. Journal of measurement technology, 2007 (8).

[4] YueKui. Automatic processing machine tool guide rail straightness error [J]. Journal of mechanical manufacturing, 2005, 43 (10).

[5] yong-chao zhang, et al. Straightness error evaluation method is briefly [J]. Modern machinery, 2005 (4). 